\title{
A Core Ontological Model for Semantic Sensor Web Infrastructures
}

\author{
Raúl Garcia-Castro, Universidad Politécnica de Madrid, Spain \\ Oscar Corcho, Universidad Politécnica de Madrid, Spain \\ Chris Hill, University of Southampton, UK
}

\begin{abstract}
Semantic Sensor Web infrastructures use ontology-based models to represent the data that they manage; however, up to now, these ontological models do not allow representing all the characteristics of distributed, heterogeneous, and web-accessible sensor data. This paper describes a core ontological model for Semantic Sensor Web infrastructures that covers these characteristics and that has been built with a focus on reusability. This ontological model is composed of different modules that deal, on the one hand, with infrastructure data and, on the other hand, with data from a specific domain, that is, the coastal flood emergency planning domain. The paper also presents a set of guidelines, followed during the ontological model development, to satisfy a common set of requirements related to modelling domain-specific features of interest and properties. In addition, the paper includes the results obtained after an exhaustive evaluation of the developed ontologies along different aspects (i.e., vocabulary, syntax, structure, semantics, representation, and context).
\end{abstract}

Keywords: Flood Emengency Planning, Ontology, Ontology Evaluation, Semantic Sensor Network Ontology, Semantic Sensor Web, Sensor

\section{INTRODUCTION}

Built upon the huge quantities of live and archived sensor data available in the Web, the so-called Sensor Web (Botts, Percivall, Reed, \& Davidson, 2006), the Semantic Sensor Web is a framework for providing enhanced meaning for sensor observations so as to increase interoperability (Sheth, Henson, \& Sahoo, 2008). Such meaning is represented through

DOI: 10.4018 /jswis.2012010102 ontologies (i.e., machine-processable formal models) that support data description, discovery and integration.

The inclusion of ontologies into Sensor Web infrastructures has led these infrastructures to evolve into Semantic Sensor Web ones, i.e., software infrastructures capable of processing sensor data that are modeled with ontologies and distributed on the Web.

The UML models designed by the Sensor Web Enablement Working Group (OGC, n.d.) in the Open Geospatial Consortium (OGC) 
(e.g., the Observations and Measurements (Cox et al., 2010) and the Sensor Model Language (Botts \& Robin, 2007)) are frequently used in sensing infrastructures and have sometimes been transformed into ontological models. Furthermore, the recent development of the W3C Semantic SensorNetwork (SSN)ontology (Lefort et al., 2011) will push forward the use of commonontological models for representing sensor networks.

However, current Semantic Sensor Web infrastructures have further representational requirements than just modelling sensor networks. This is because sensor data are distributed, which requires being aware of the different sensor datasets available; are heterogeneous, which requires knowing the schemas used in these datasets; and are web-accessible, which requires having descriptions of the web services that provide dataset access.

Nevertheless, none of the data models proposed fully covers these requirements; they are partially covered in some models but some requirements have not been dealt with yet (i.e., dataset schema descriptions).

Furthermore, in addition to the types of data just mentioned, Semantic Sensor Web infrastructures need to manage further data (e.g., geographical, organizational, or domainspecific data); thus, one of the dilemmas that developers face is to know which is the most appropriate way to model domain-specific features of interest and their properties, so that they can link domain data with sensor data.

The main contribution of this paper is the definition of a core ontological model for Semantic Sensor Web infrastructures that allows describing sensor networks (by extending the SSN ontology), sensor data sources with their underlying schemas, and the web services that these sources expose.

This core ontological model provides a shared vocabulary to discover, access, and integrate information within the infrastructure and to interoperate both across the internal infrastructure services and between an infrastructure and the external sources that adopt alternative approaches (e.g., the OGC Sensor Web Enablement ones) (Botts et al., 2006).

A second contribution is a set of guidelines to model domain-specific features of interest and properties; these guidelines are based on frequent requirements and, in contrast to the current practice, they encourage to take advantage of the expressiveness of the underlying logical formalism and to add logical constraints to the model.

As an example of application, the core ontological model is currently being used in a Semantic Sensor Web infrastructure that is being applied to a use case in the domain of coastal flood emergency planning (Gray et al., 2011). This development required us to define a set of ontologies to represent those data that are specific to that domain, and to this end the mentioned guidelines have been applied.

As any other engineered product, ontologies may have defects; some of these defects may appear during their development, but others only emerge once they have been deployed. The ontologies described in this paper have been evaluated through different aspects (i.e., vocabulary, syntax, structure, semantics, representation, and context) to ensure their suitability for their intended use scenario; in this paper we present the details of such an evaluation to guide future evaluation efforts.

This paper is structured as follows. First, it presents the use case in the coastal flood emergency planning domain where the ontologies have been used. Second, it gives an overview of the ontological model and introduces the different modules that compose it. Then it describes the ontology modules that deal with infrastructure data, that is, an extension of the SSN ontology and modules for representing web services, datasets, and dataset schemas. The following section presents the guidelines to model domain specific features of interest and properties along with the domain-specific ontology modules. Then, it describes the evaluation performed over the ontological model and compares our model with other models used in sensing infrastructures. Finally, it provides some conclusions derived from this work. 


\section{Coastal Flood Emergency Planning Use Case}

This section describes a use case in the coastal flood emergency planning domain that has been resulted in a web application (http://www.semsorgrid4env.eu/services/dynamic-demo) built on top of a Semantic Sensor Web infrastructure (Gray et al., 2011). This application, on the one hand, integrates data from a wide variety of sources and supports their consumption and, on the other hand, requires modelling and processing heterogeneous data from different domains. Therefore, it can be considered as an exemplar of mash-up that can be developed on top of Semantic Sensor Web infrastructures.

The use case scenario takes place in the Solent region in the south coast of England, which has a complex tidal and wave pattern that generates a demand for sea-state forecasts from different types of users (e.g., emergency response planners, harbour masters, members of the public).

Once logged into the emergency planning web application, the application provides an initial characterization of the data that is relevant to the user, depending on his/her role. Besides, the user can discover other relevant data sources based on their content. Once the user selects which data sources to show, the application juxtaposes such sources as layers on a map that the user can dy namically browse.

The representational requirements of the application mainly involve modelling domainspecific information (concrete features of interest and properties in the coastal flood domain, relevant regions of the south coast of England, and the different user roles) and relating this information to observations coming from sensor networks.

The underlying Semantic Sensor Web infrastructure has to manage and integrate data coming from different heterogeneous data sources with a diverse ty pology: relational data, streaming data, and data produced by environmental models.

Streaming data consist on wave, tide and meteorological information coming from two different sensor networks (each covering the three data categories) that have been deployed by the Associated British Ports authority (http://www.abports.co.uk/) and by the Channel Coastal Observatory (CCO) (http://www. channelcoast.org/).

Relational data consist on information related to the $\mathrm{CCO}$ sensor network, on the archive of the CCO sensor observations, and on information about flood defences coming from the UK Environment Agency's National Flood and Coastal Defences database (http:// www.scisys.co.uk/casestudies/).

Three environmental models, using the previous data, provide forecasts about the sea condition (wave height and sea level), defence over-topping (probability of defences being over-topped), and flood levels (water depth on flood plains in the event of over-topping).

Furthermore, the application uses other data sources external to the infrastructure: weather forecasts from public meteorological web services, details of shipping in the region obtainedfrom the RDF (Klyne \& Carroll, 2004) data published by the Automatic Identification System network (United States Department of Homeland Security, n.d.), and public traffic reports from an RSS feed (RSS Advisory Board, 2009).

The infrastructure also allows creating virtual data sources by integrating data from heterogeneous sources, both in terms of the modality of data (i.e., streaming and relational) and of the representation of data(i.e., the schema used); this integration requires to have information about the different types of data and their corresponding schemas.

All the data sources used in the infrastructure are published in the Web through web services, some of them internal to the infrastructure and some external; therefore, the infrastructure has to store the semantic descriptions of these services.

\section{OVERVIEW OF THE ONTOLOGICAL MODEL}

This section presents an overview of the core ontological model for Semantic Sensor Web 
Figure 1. Overview of the ontological model

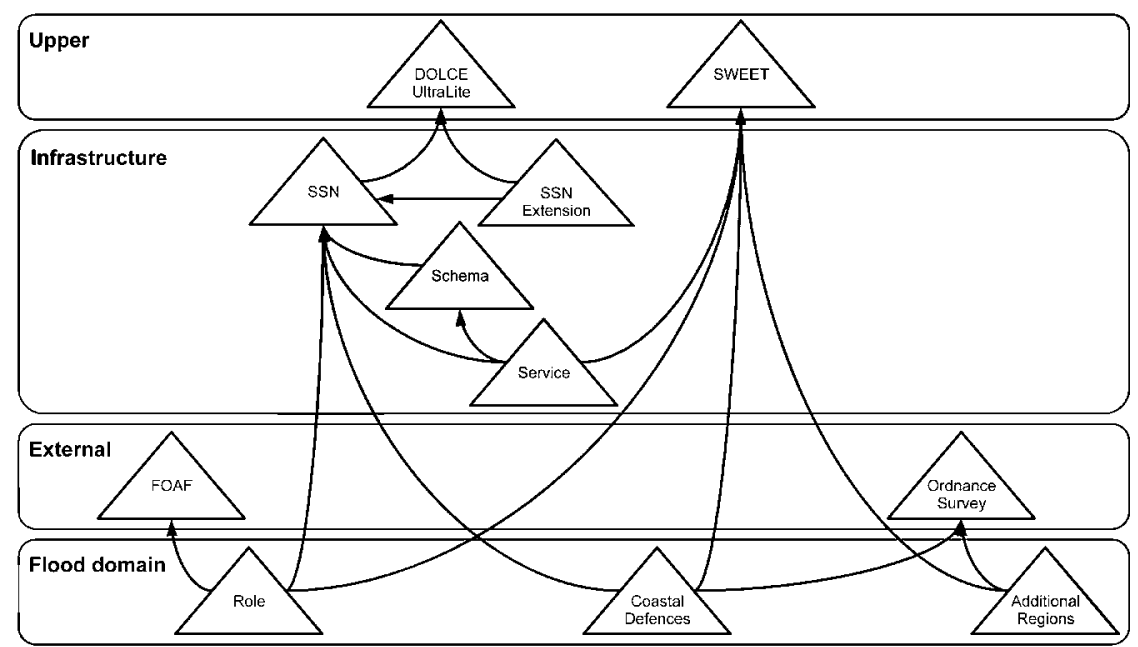

infrastructures as well as the ontologies developed to support the use case described in the previous section. All the ontologies that compose this model have been implemented in OWL (McGuiness \& van Harmelen, 2004) and are publicly available (Gómez-Pérez, Martinez, Fernandes, \& Ibarrola, 2008).

Figure 1 illustrates how the ontologies in the ontological model can be classified in different layers according to whether the ontology represents domain-specific information, information required for the infrastructure or upper-level information to facilitate interoperability among the other ontologies.

These ontologies satisfy different knowledge representation requirements extracted during the development of the infrastructure and the use case prototype. These requirements are

- To represent sensor networks and their observed information about properties of certain features of interest. This requirement is covered by the SSN ontology, developed by the W3C Semantic Sensor Network Incubator Group (Lefort, Sheth, \& Taylor, 2008), and by the SSN Extension ontology. These ontologies reuse the
DOLCE+DnS UltraLite upper ontology (OGC, n.d.).

- To represent schemas of relational databases and relational streams. This requirement is covered by the Schema module that extends and corrects an ontology for relational data and schema components (Pérez de Laborda \& Conrad, 2005).

- To represent the web services provided by the infrastructure and the datasets they give access to. This requirement is covered by the Service module that reuses the SWEET upperontologies (Raskin \&Pan, 2005) and includes concepts from the ISO19119 standard on geographic information services (ISO/IEC, 2005b).

- To represent those features of interest and their properties specific to the coastal flood emergency planning use case. This requirement is covered by the Coastal Defences ontology.

- To represent the geographic and administrative regions of the south coast of England. This requirement is covered by the Ordnance Survey ontologies (http:// www.ordnancesurvey.co.uk/oswebsite/ ontology/), which include the regions of Great Britain, and by the Additional 
Figure 2. Overview of the SSN ontology (Lefort et al., 2011) (O 2011, W3C, MIT, ERCIM, Keio, Used with permission)

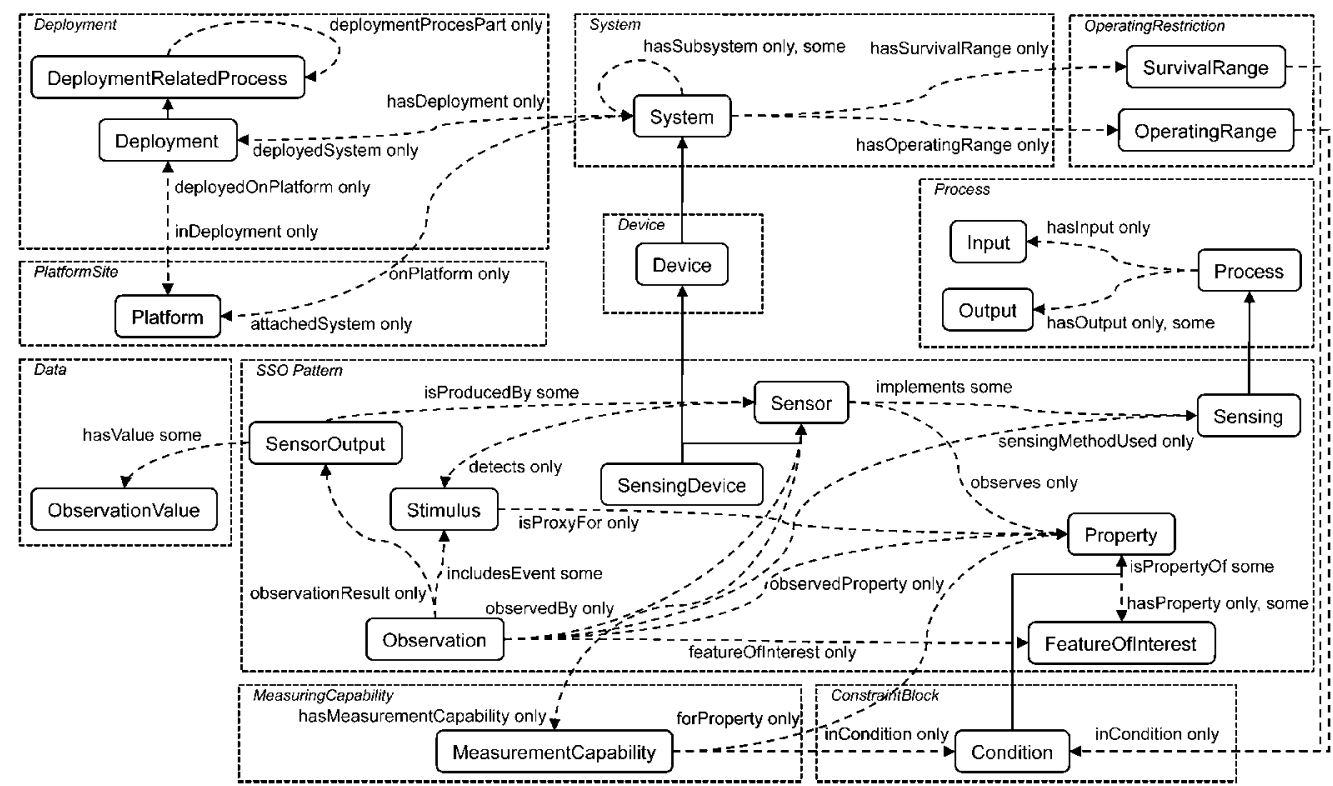

Regions ontology, which includes other regions needed in our use case.

- To represent the different roles involved in the use case. This requirement is covered by the Role ontology.

The following sections describe each of these ontologies. When naming classes or properties, the namespace of the class or property will be used if they belong to an external ontology (c.g., sw:Dataset).

\section{INFRASTRUCTURE ONTOLOGIES}

This section describes those ontology modules that deal with Semantic Sensor Web infrastructure data, that is, sensor observations and descriptions of datasets, dataset schemas, and web services.

\section{Extending the Semantic Sensor Network Ontology}

The Semantic Sensor Network ontology (SSN) (http://purl.oclc.org/NET/ssnx/ssn) (Lefort et al., 2011) revolves around several conceptual modules that encompass key sensor concepts. The relationships between these ontology modules appear in Figure 2, which contains an overview of the main classes and properties inside these modules.

The modules can be used to represent particular aspects of a sensor orits observations, for example, sensors, observations, features of interest, the process of sensing (i.e., how a sensor operates and observes), the deployment of sensors or their attachment to platforms, the measuring capabilities of sensors, as well as their environmental and survival properties in specific environments. 
Figure 3. Extensions to the SSN ontology

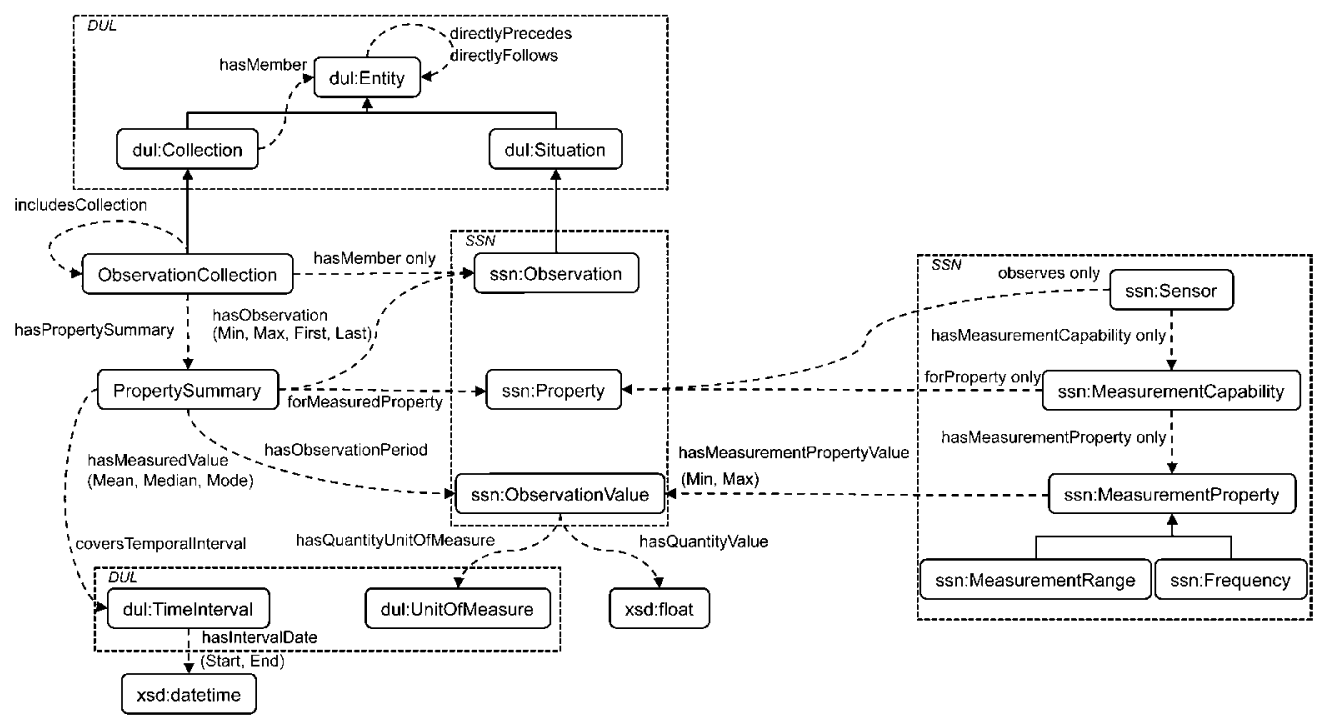

The main classes of the SSN ontology have been aligned with classes in the DOLCE+DnS Ultra Lite foundational ontology to facilitate reuse and interoperability.

The SSN ontology is intended to be refined and extended in specific use scenarios, such as ours. In our case, we required to model some informationabout sensor networks not covered by the SSN ontology. Such information, shown in Figure 3, is the following:

- Observation collections (i.e., sets of observations sharing some characteristic) that, even if they are defined in the OGC Observations and Measurements specification (Cox et al., 2010), were not included in the SSN ontologies.

- Property summaries for observation collections that contain, for a certain property, relevant pieces of information(e.g., the first observation, the temporal interval covered, or the maximum measured value).

- Units of measurement for observation values since the SSN ontology imposes no restrictions to represent them.

- Measurement property values in a way similar to that proposed in the examples of the SSN Incubator Group report (Lefort et al., 2011).

For extending the SSN ontology we chose to reuse the DOLCE+DnS Ultra Lite ontology because it is the ontology reused by SSN, even if there may be other alternatives to represent some of the concepts needed (e.g., the Measurement Units Ontology for measurement units) (Berrueta \& Polo, 2008).

\section{Schema Ontology}

The Schema ontology is used to represent schemas of relational databases and relational streams. For the development of this ontology, an ontology for relational data and schema components (Pérez de Laborda \& Conrad, 2005) was taken as a starting point and adapted to our case.

The mainconcepts of the Schema ontology, which is depicted in Figure 4, are schemas, their extents, and extent attributes. These schema descriptions and their relationships with observed properties allow exploiting schema information in data integration tasks. Next, we present in detail how these concepts can be described. 
Figure 4. Overview of the schema ontology

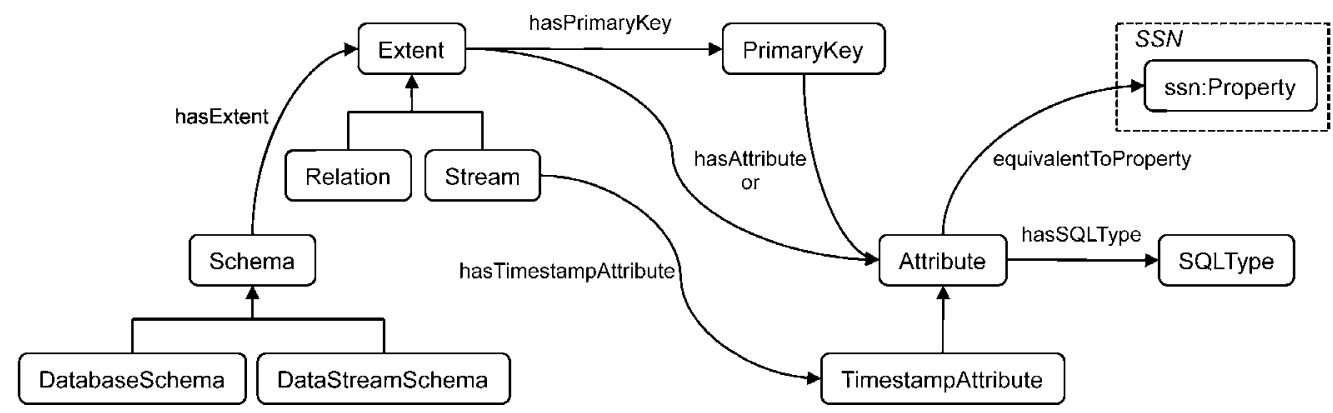

A schema, which can be either a database schema or a data stream schema, is described with its extent that, in turn, can be a relation or a stream. Extents can have primary keys and both the extents and the primary keys can have attributes.

Attributes are defined by means of their SQL type (we use the SQL-92 datatypes) (Digital Equipment Corporation, 1992) and of the WebRowSet metadata attributes (Oracle, 2011). Besides, in order to facilitate the mapping of attributes, attributes are related to the property they are equivalent to.

Finally, streams have a timestamp attribute, which is a special type of attribute that has a Timestamp SQL type.

\section{Service Ontology}

The Service ontology is used to represent the web services provided by the sensing infrastructure and the datasets they provide access to.

The main concepts of the Service ontology, depicted in Figure 5, are web services, their interfaces, and the datasets that the web services expose. Next, we provide details of how these concepts can be described.

A web service is described according to the endpoint reference used to access it, its interface, the dataset that it exposes, and its service type. Service types define, on the one hand, the different service types that are part of the infrastructure and, on the other hand, a classification of services according to the representation format returned by the service, e.g.,
XML, RDF, RSS, GeoJSON, or some OGC specification (SOS, SPS, SAS, WNS, CSW, WFS, and WMS). The ontology also allows specifying whether a web service is a stateful web service.

A web service interface is described by the operations that it contains and by the parameters of these operations; these three concepts have been extracted from the ISO19119 standard on geographic information services (ISO/IEC, 2005b). The ontology allows distinguishing between different types of interfaces, i.e., data access, integration, notification, pull point, query, service, subscription, and subscription manager interfaces.

A dataset is described according to the temporal extent and the region (and, correspondingly, the spatial extent of such region) that it covers; all these classes are reused from the SWEET ontologies (Raskin \& Pan, 2005). A dataset is also described by the features of interest and properties for which it includes data (reusing the respective classes from the SSN ontology), by the schema that is has (reusing the Schema ontology), and by the URLs that contain information about the visualisation styles to be used with the dataset.

\section{DOMAIN ONTOLOGIES}

This section starts by presenting some guidelines to model domain-specific features of interest and properties. Then, it describes the ontology modules that deal with data in the domain of 
Figure 5. Overview of the service ontology

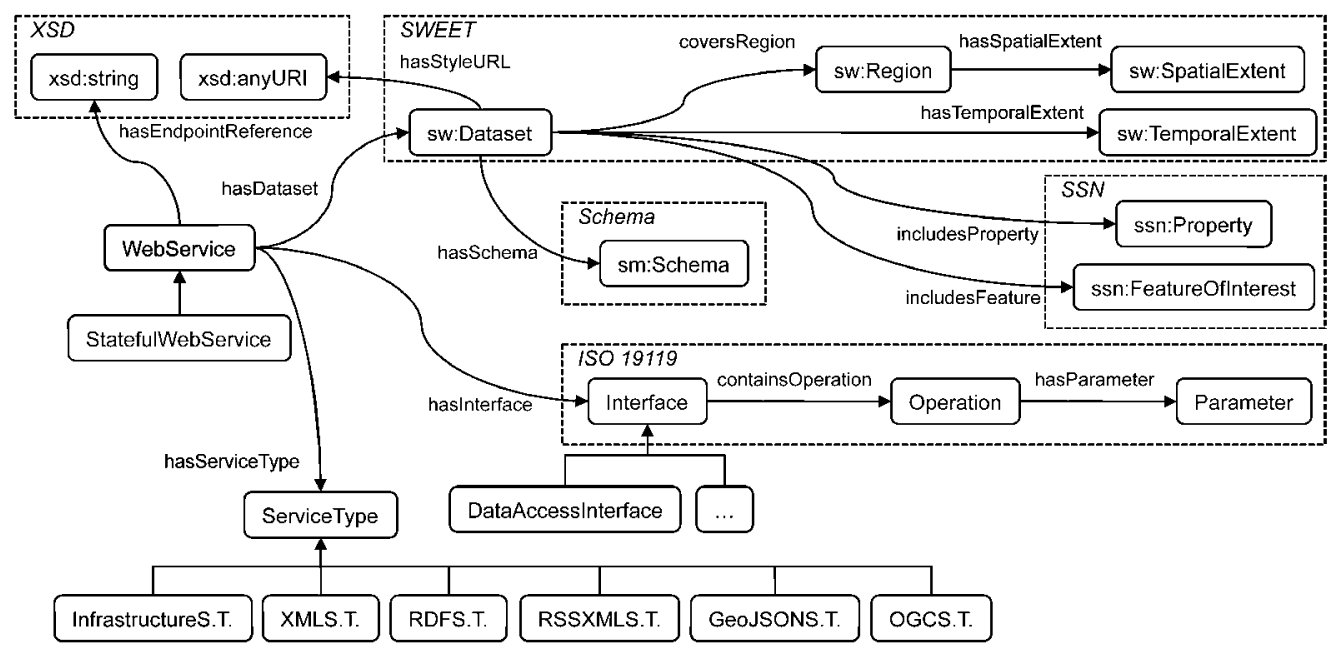

our use case (i.e., coastal flood emergency planning) that have been developed following those guidelines.

\section{Modelling Domain Features and Properties}

The two main types of infrastructure data that require to be linked to domain-specific features of interest and properties are dataset descriptions, in which a dataset and the attributes of its underlying schema are related to certain features and properties, and observation data, in which observation values (regardless of their source) are related to the features and properties observed.

Next, we provide some guidelines to satisfy a common set of requirements related to modelling domain-specific features of interest and properties so they both canbe used inthese data. It should be added that the two first requirements always appear, while the other two may appear or not depending on the specific use case.

\section{Modelling features of interest and feature} categories. The common approach for modelling features of interest and feature categories is to create a class or a class hierarchy of feature categories (e.g.,
Room). A concrete feature of interest will be an instance of one of the classes (e.g., room 102). This is the notion followed in the Observations and Measurements specification (Cox et al., 2010) and adopted in those developments derived from it (McCarthy, Graniero, \& Rozic, 2008; Bartha et al., 2009; Moodley, 2009; Lefort et al., 2011).

Modelling properties and property categories. Similarly to the previous case, and also widely adopted, the approach for modelling properties and property categories is to create a class or a class hierarchy of property categories (e.g., RoomProperty). A concrete property will be an instance of one of the classes (e.g., temperature). Care must be taken to avoid problems in the use of properties and their categories; for example, in Moodley (2009) an observation is linked with a property named pressure 1 that is an instance of a class named Pressure. This approach may cause problems if the class is used in the data instead of the instance or if the class has multiple instances that relate to the same property (e.g., pressure 1 and pressure 2).

Linking features of interest and properties. Even if features of interest are usually related to their properties through the observa- 
Figure 6. Excerpt of the coastal defences ontology

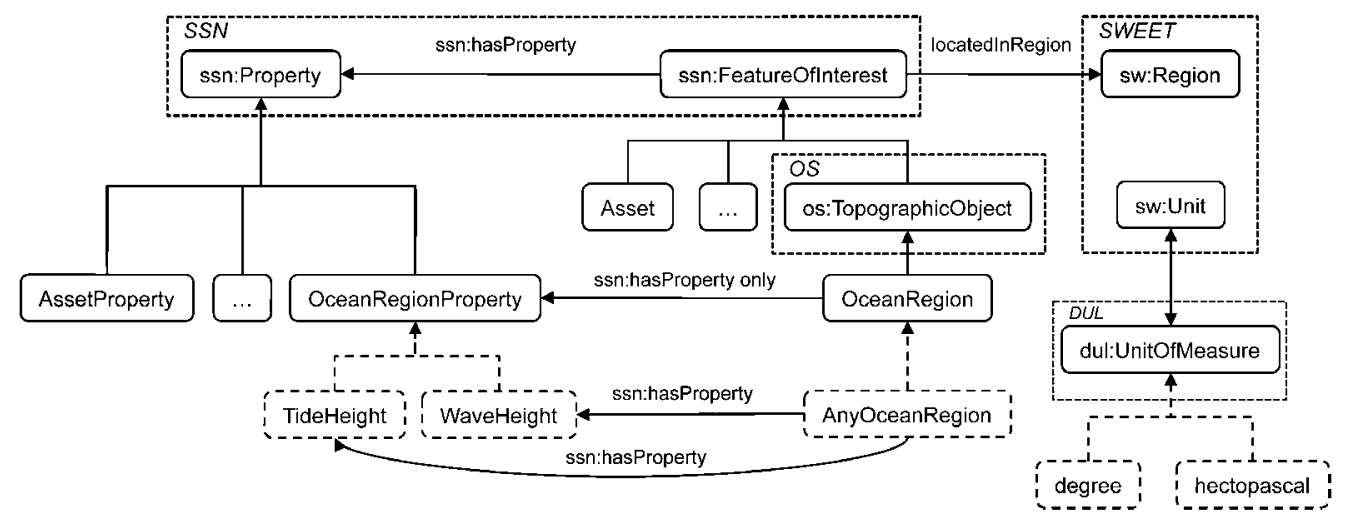

tions that contain them, some use cases require these entities to be directly linked (e.g., to manage them without accessing observation data). Using the SSN ontology this linkage can be done by means of the hasProperty and isPropertyOf properties; however, we advise to add existential value constraints (i.e., owl:someValuesFrom) to the feature category classes in order to restrict the properties that a certain feature category can have.

Linking feature categories and properties. In order to obtain information about feature categories (e.g., which properties describe a certain feature category, or which feature categories are covered in a certain dataset) we need to query all the observation data that, besides, may not be complete. One solution to enable answering questions like the previous ones without dealing with observation data is to define generic features of interest. This definition can be done by creating, foreach feature category class, an instance that represents a fictitious generic individual of that class (e.g., anyRoom) and by relating that instance to all the existing properties for that feature type.

To create the classes and individuals mentioned, existing entities should be reused as much as possible, either from domain ontologies or from upper ontologies (e.g., DOLCE or SWEET). In addition, it is not advisable to directly use classes in the data instead of their instances (e.g., Room hasProperty temperature) because those classes then become metaclasses (i.e., classes that are both a class and an instance) and may cause reasoning problems.

\section{Coastal Defences Ontology}

The Coastal Defences ontology, depicted in Figure 6, is used to represent those features of interest and their properties that are specific to the coastal flood emergency planning use case.

As it can be observed, features of interest are located in a certain region, and the ontology defines a hierarchy with the categories of features of interest that are relevant to our use case (e.g., ocean regions). This modelling approach is the one proposed in the ISO 19109 General Feature Model (ISO/IEC, 2005a).

For each feature category, as established in the General Feature Model, the ontology defines the properties it can have by means of a property category (e.g., ocean region properties) and one individual for each property in that category (e.g., tide height or wave height). In our case we also add one generic feature related to all the properties in that category (e.g., a generic ocean).

The current version of the Coastal Defences ontology contains properties for the following features of interest: physical atmosphere, asset, 
Figure 7. Overview of the role ontology

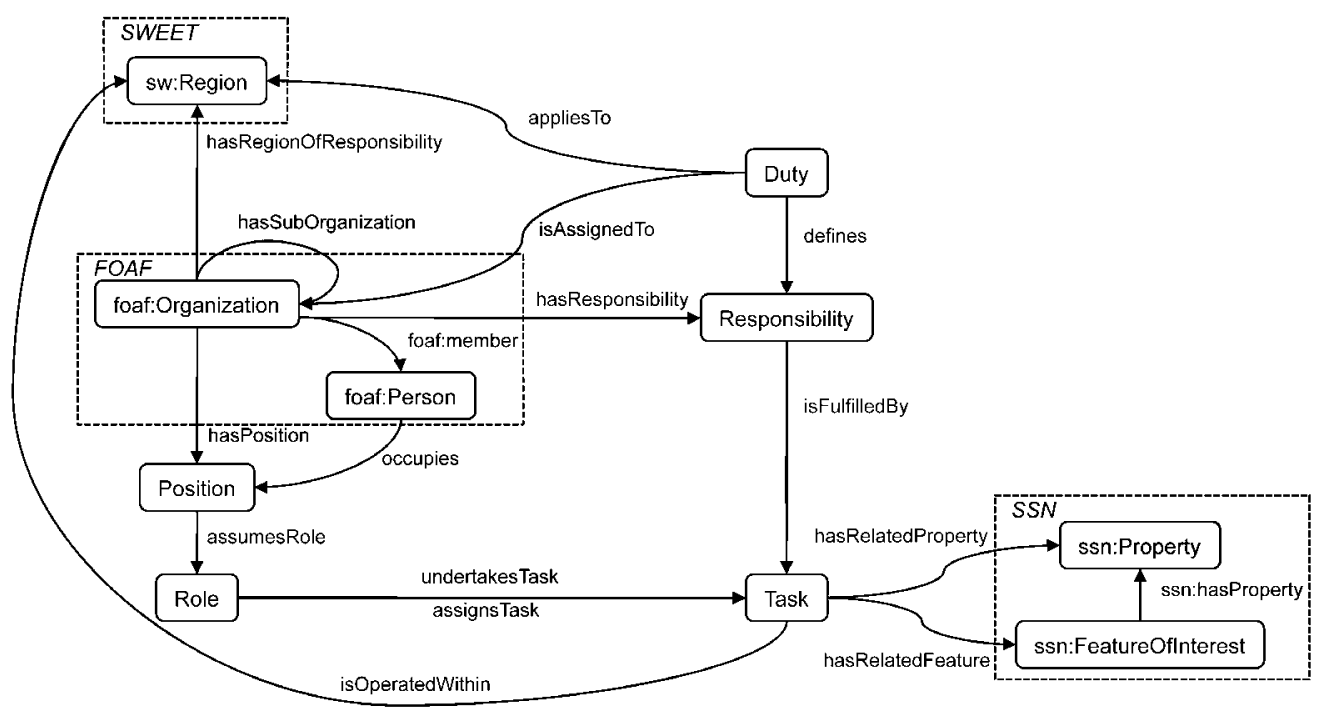

flood plain, flood zone, flood defence policy, Role Ontology ocean region, vessel, and road problem.

The ontology also defines some units of measurement needed in the use case not described in the SWEET ontologies (i.e., degree and hectopascal).

\section{Additional Regions Ontology}

The Additional Regions ontology is used to represent those regions of the south coast of England that are relevant to the use case but that are not covered by the Ordnance Survey ontologies.

The need for additional regions occurs eitherbecause specific regions are not included in the Ordnance Survey ontologies (e.g., the Coastal Defence Partnership, which is composed of Gosport, Havant and Portsmouth) or because different services provide different boundaries for the same region (e.g., the boundaries of South East England for the Channel Coastal Observatory are different from those for the BRANCH).

The Role ontology is used to represent the different roles involved in a coastal flood emergency planning scenario and enables a role-based customization of user interfaces and data.

The main concepts of the Role ontology, depicted in Figure 7, are duties, organizations and roles. Next, we provide details of how these concepts can be described.

A main concept in the ontology is that of duty (e.g., the Flood and Water Management Act, 2010). A duty applies to a certain region, is assigned to an organization and defines the set of responsibilities for such organization (e.g., flood coastal erosion risk management). These responsibilities are fulfilled by a set of tasks, which are related to certain features of interest and properties.

Furthermore, an organization has some regions of responsibility and can have suborganizations, each with different regions of responsibility. Additionally, an organization has a number of positions that operate within the 
corresponding region and different members that occupy those positions. Each position in the organization assumes a specific role that defines the different tasks to be undertaken by that role.

\section{EVALUATION OF THE ONTOLOGIES}

This section presents how we have evaluated the ontologies and the results obtained. Our use case imposed no ontology quality requirements other than conformance to the Semantic Web standards and to the Linked Data principles (Berners-Lee, 2006) since the Semantic Sensor Web infrastructure is intended to publish sensor data on the Web, in the form of linked sensor data (Page, De Roure, Martinez, Sadler, \& Kit, 2009).

In order to evaluate any ontology, we need to know the different aspects of the ontology to be evaluated, the techniques to be used to evaluate these aspects, and the reference frame to compare to and draw conclusions.

Multiple ontology evaluation aspects and perspectives have been identified in different ontology evaluation frameworks based on the description of ontology quality characteristics (Gangemi, Catenacci, Ciaramita, \& Lehmann, 2005), on literature reviews (Brank, Grobelnik, \& Mladenic, 2005), on existing evaluation studies (Obrst et al., 2007), or on a holistic view of evaluation (Pak \& Zhou, 2010). However, these frameworks are mostly theoretical and do neither include a set of concrete evaluation techniques to be used, even if they include references to existing work, nor any comparison reference or baseline.

Inthis work, we have followed the ontology evaluation framework proposed by Vrandečić (2010). This framework establishes the different aspects of an ontology that can be evaluated, comprehensively subsuming previous work. Besides, it proposes different evaluation methods that can be applied for each aspect; these methods either consist of checks to be performed over the ontology or of metrics that can be compared against a set of constraints or against reference values extracted from an ontology corpus.

These reference values allow us to compare our ontology with other ontologies published in the Web that cover different domains. This is useful in our case since we cannot check whether the ontology satisfies concrete ontology quality requirements, which do not exist in our case, or perform a quantitative comparison with other ontologies or models, which cannot be performed both because their schema definitions are not available and because models have different modelling requirements and, therefore, different domain coverage.

The ontology aspects taken into account in the evaluation framework are the following:

- Vocabulary. Evaluating the vocabulary aspect of an ontology means to evaluate the names used in the ontology. Names can be URI references or literals, and this aspect deals with the different choices available regarding naming entities or leaving entities unnamed (i.e., blank nodes).

- Syntax. This aspect deals with evaluating the syntax in which the ontology is serialised. Ontologies can be described in a number of different surface syntaxes; often syntactic descriptions within a certain syntax can differ widely.

- Structure. Since an ontology can be described by an RDF graph, this aspect deals with the evaluation of the structure of such graph. The structure can vary highly even describing semantically the same ontology.

- Semantics. This aspect deals with the evaluation of the formal meaning of an ontology through different metrics that go beyond the structure of the ontology and exploit its semantics.

- Representation. This aspect captures the relation between the structure and the semantics. Representational aspects are usually evaluated by comparing metrics calculated on the RDF graph with features of the possible models as specified by the ontology. 
- Context. This aspect is about the features of the ontology when compared with other artefacts in its environment; e.g., a data source that the ontology describes, a different representation of the data within the ontology, or formalised requirements for the ontology in form of competency questions or additional semantic constraints.

The next sections present how the ontologies were evaluated according to each of the aspects.

\section{Vocabulary Evaluation}

The evaluation methods proposed for this aspect mainly consisted in checking certain characteristics related to the URI syntax (correctness and protocols used), the HTTP protocol (response codes and content types), naming and annotation (conventions, labels, comments, and language tags), and entity definition (declarations, datatypes, and superfluous blank nodes).

We did not expect to find major issues in this aspect because we used a common naming and annotation scheme during the ontology development, and added labels, comments and language tags (i.e., "en") where needed. The only defects found were the following.

While checking the HTTP status codes of the URIs used in the ontologies (by making GET calls to them), we noticed that two URIs from the Ordnance Survey (OS) ontologies returned a 404 (Not Found) error; this error was caused because the OS ontologies had changed, and the references to the URIs of the old ontology versions could not be resolved anymore. Once the ontologies were updated to the last version of the OS ontologies, the problems with these two URIs disappeared.

While checking whether the type of every URI was declared (as an OWL class, individual, datatype, object or annotation property), we found that in two ontologies not every URI was declared; in the Coastal Defences ontology, the three individuals that are reused from the SWEET units of measurement ontology and in the Schema ontology, all the individuals that define the SQLType enumerated class. Even though it is straightforward to identify these URIs as individuals (either by a person or by a reasoner), we added the declarations for those URIs so as to avoid having OWLFull ontologies.

Besides these checks, different ontology reuse metrics are proposed in Vrandečić (2010), which are the following:

- Number of namespaces used in the ontology $\left(N_{N S}\right)$.

- Number of unique URIs used in the ontology $\left(N_{U N}\right)$.

- Number of URI name references used in the ontology (every mention of a URI counts) $\left(N_{N}\right)$.

- Ratio of name references to unique names $\left(R_{N U}=\frac{N_{U N}}{N_{N}}\right)$.

- Ratio of unique URIs to namespaces $\left(R_{U N S}=\frac{N_{U N}}{N_{N S}}\right)$.

Table 1 shows the reuse metrics obtained from the ontologies. It does not include those names and namespaces that are part of the $\mathrm{RDF}(\mathrm{S})$ or OWL vocabularies.

Compared to the results obtained in Vrandečić (2010) for the ontologies in the Watson EA corpus (a subset of the Watson corpus that is part of the Billion Triple Challenge data) (Maynard \& Harth, 2012), it can be observed that the values of $R_{N U}$ are consistent with those of the ontologies in the corpus (lower than 0.5), whereas the values of $R_{U N S}$ and $N_{N S}$ are not consistent since they are expected to be lower than 5 and greater than 10 , respectively.

With regard to the ratio of unique URIs to namespaces, to expect less than five unique URIs per namespace is to expect that ontologies have a low number of entities (five for an ontology with one namespace, ten for an ontology with two namespaces, and so on). Taking into account the number of namespaces, to expect that ontologies include more than ten 
Table 1. Reuse metrics for the ontologies

\begin{tabular}{|l|l|l|l|l|l|}
\hline \multicolumn{1}{|c|}{ Ontology } & \multicolumn{1}{c|}{$N_{N S}$} & \multicolumn{1}{c|}{$N_{U N}$} & \multicolumn{1}{c|}{$N_{N}$} & \multicolumn{1}{c|}{$R_{N N}$} & \multicolumn{1}{c|}{$R_{U N S}$} \\
\hline Additional Regions & 5 & 26 & 100 & 0.26 & 5.2 \\
\hline Coastal Defences & 8 & 94 & 503 & 0.19 & 11.75 \\
\hline Role & 4 & 26 & 113 & 0.23 & 6.5 \\
\hline Schema & 2 & 65 & 293 & 0.22 & 32.5 \\
\hline Service & 7 & 56 & 189 & 0.30 & 8 \\
\hline SSN Extension & 3 & 33 & 130 & 0.25 & 11 \\
\hline
\end{tabular}

namespaces is to expect a significant reuse in ontologies.

One issue here can be the way in which the expected values were calculated; this is not clearin Vrandečić (2010) and could significantly affect the values of the metrics. For example, if we create an empty ontology with the Protégé ontology editor, the editor already creates five namespaces in the RDF/XML serialisation(i.e., $r d f, r d f s, o w l, x s d$, and $o w l 2 x m l)$. As mentioned, we did not count these namespaces in our metrics, but if we added five more namespaces to each ontology the values would be similar to the expected ones.

\section{Syntax Evaluation}

The evaluations regarding the syntaxaspect were performed over the RDF/XML serialisations of the ontologies. These evaluations were intended to check out problems with the serialisations with the RDF Validator (Prud'hommeaux, 2007) and the OWL Validator (Bechhofer \& Volz, 2003) and to verify if the character encoding of the serialisations is UTF-8. No problems in this aspect were found, as expected, since an ontology editor was used during the ontology development (i.e., Protégé).

\section{Structure Evaluation}

The evaluation methods proposed for this aspect consisted in checking the ontology complexity and searching for problems in the ontology using anti-patterns and the OntoClean method.

\section{Ontology Complexity Metrics}

We counted how many times each ontology language feature appeared in the ontologies. This feature analysis allowed us to calculate the complexity of the language fragment used in each ontology by means of anonline complexity calculation tool (Zolin, 2011).

Table 2 shows the structural metrics obtained (only those features that appear at least in one ontology are shown), the DL expressiveness, the OWL species, and the reasoning complexity for each ontology ${ }^{1}$. As can be seen, most of the ontologies have Pspace complexity except the SSN Extension ontology that has ExpTime complexity.

\section{Anti-Patterns}

We executed two SPARQL queries over the ontologies to discover potentially problematic patterns. The two queries executed were those proposed in Vrandečić (2010) for detecting the anti-pattern of subsuming nothing and for detecting the anti-pattern of skewed partitions. None of these anti-patterns were found in the ontologies.

\section{OntoClean}

We used the OntoClean method (Guarino \& Welty, 2002) to detect subsumption misuses in the ontologies. For the detection, we tagged every class in an ontology with certain metaproperties (i.e., rigidity, unity, dependence, 
Table 2. Structural metrics for the ontologies

\begin{tabular}{|c|c|c|c|c|c|c|}
\hline Metrics & $\mathbf{A R}$ & CD & RO & SM & so & SS \\
\hline Class & 2 & 35 & 10 & 11 & 27 & 9 \\
\hline Object property & 1 & 4 & 15 & 6 & 11 & 19 \\
\hline Data property & & & & 19 & 2 & 4 \\
\hline Individual & 22 & 53 & & 28 & 15 & \\
\hline \multicolumn{7}{|l|}{ Class Axioms } \\
\hline Sub-class & & 35 & & 6 & 15 & 2 \\
\hline Equivalent class & & 1 & & 1 & & \\
\hline \multicolumn{7}{|l|}{ Object property axioms } \\
\hline Sub-object property & & & & & & 9 \\
\hline Functional object property & & & & 2 & & \\
\hline Object property domain & & 1 & 15 & 6 & 11 & 9 \\
\hline Object property range & & 1 & 15 & 6 & 11 & 9 \\
\hline \multicolumn{7}{|l|}{ Data property axioms } \\
\hline Sub-data property & & & & & & 2 \\
\hline Data property domain & & & & 19 & 2 & 2 \\
\hline Data property range & & & & 19 & 2 & 2 \\
\hline \multicolumn{7}{|l|}{ Individual axioms } \\
\hline Class assertion & 22 & 50 & & & 15 & \\
\hline Object property assertion & 10 & 42 & & & & \\
\hline \multicolumn{7}{|l|}{ Annotation axioms } \\
\hline Entity annotation & 22 & 151 & 25 & 45 & 36 & 26 \\
\hline DL expressiveness & $\mathrm{AL}$ & $\mathrm{AL}$ & $\mathrm{AL}$ & ALCOF(D) & $\mathrm{AL}(\mathrm{D})$ & $\mathrm{ALH}(\mathrm{D})$ \\
\hline OWL Species & Lite & Lite & Lite & DL & Lite & Lite \\
\hline Complexity & Pspace & Pspace & Pspace & Pspace & Pspace & ExpTime \\
\hline
\end{tabular}

and identity) and then checked whether a set of subsumption constraints held.

We applied the OntoClean method manually to the ontologies, since their number is low and there are few subsumptions in them. While applying the method, no problem was found in the ontologies.

\section{Semantics Evaluation}

The evaluation performed consisted in comparing the normalised class depth measure of the ontologies (i.e., the length of the longest subsumption path of the normalised version of the ontology) with the stable minimal depth of the ontology (which takes the open world assumption into account) (Vrandečić, 2010).

If these two values are the same there is no problem with the ontology, which is the case of our ontologies that include class hierarchies.

\section{Representation Evaluation}

The evaluation methods proposed for this aspect consist in calculating a set of ontological metrics and checking whether a set of constraints hold for them. These metrics are the following: 
Table 3. Ontological metrics for the ontologies

\begin{tabular}{|l|l|l|l|}
\hline \multicolumn{1}{|c|}{ Ontology } & \multicolumn{1}{c|}{$(E T(O))$} & \multicolumn{1}{c|}{$\left(R_{c}(O)\right)$} & \multicolumn{1}{c|}{$\left(R_{p}(O)\right)$} \\
\hline Additional Regions & - & 1 & 1 \\
\hline Coastal Defences & 1 & 1 & 1 \\
\hline Role & - & 1 & 1 \\
\hline Schema & 1 & 1.09 & 1 \\
\hline Service & 1 & 1 & 1 \\
\hline SSN Extension & 1 & 1 & 1 \\
\hline
\end{tabular}

- Explicitness of the subsumption hierarchy $(E T(O))$, which is the ratio between the maximum depth of the taxonomy of an ontology and the maximum subsumption path length of the normalised version of the ontology.

- Ratio of classes and class names $\left(R_{C}(O)\right)$, which is the ratio between the number of classes in the normalised version of the ontology and the number of classes in the ontology.

- Ratio of properties and property names $\left(R_{p}(O)\right)$, which is the ratio between the number of properties in the normalised version of the ontology and the number of properties in the ontology.

The expected result for these three metrics is that their values are 1 (i.e., that the counts in the ontology and in its normalised version are the same) and this is the case for all the ontologies except in one case, as can be seen in Table 3 (in those ontologies without hierarchies the value of $E T(O)$ cannot be calculated).

In our case most of the values are 1 because almost every normalised ontology is identical to the original one. The only exception appears in the Schema ontology where one of the constraints from Vrandečić (2010) is not satisfied $\left(R_{C}(O)>1\right)$, which indicated "that not all interesting classes or properties have been given a name, i.e., the coverage of classes and properties with names may not be sufficient."

The constraint was not satisfied because the Schema ontology includes a complex class description (i.e., union) as domain of a property that, after normalisation, produces a new class name. It was a design decision not to include that artificial class name in the ontology, and to increase clarity we defined the domain as a union of classes. Therefore, this constraint violation entails no defect in the ontology.

\section{Context Evaluation}

For evaluating the context aspect we did not follow any of the methods proposed in Vrandečić (2010), since they rely on having a set of artefacts that we had not produced during ontology development (i.e., competency questions, test ontologies, highly-axiomatised versions of the ontology, or translation of the ontology to a logic program with rules); we thought that explicitly preparing any new artefact from the ontology to be used for the evaluation may produce biased results.

In their survey, Brank, Grobelnik, and Mladenić (2005) classify ontology evaluation approaches into comparison with a golden standard, use of the ontology in an application, comparison with a source of data, and performance of human-based evaluations.

Even if no formal evaluation has been performed in this sense, the ontologies can be considered, to a certain extent, as validated; they are being successfully used in the coastal flood emergency planning web application and have been manually inspected by the domain experts and developers that worked in the development of the prototype. 
In addition, in our case we did not have a goldenstandard on which tobase the evaluation; however, we had the data used in the prototype mentioned so we performed a data-driven analysis to calculate the completeness between an ontology and domain of knowledge (Brewster, Alani, Dasmahapatra, \& Wilks, 2004).

In the analysis we found that all those ontologies that are part of the Semantic Sensor Web infrastructure (Schema, Service and SSN Extension) were developed with the aim of their being generic and, therefore, their coverage is not high. On the other hand, those domain ontologies explicitly developed for the prototype (Additional Regions and Coastal Defences) have a high coverage because we prioritised applicability in the prototype over reusability and we modelled only all those entities that were expected to be used. Finally, the coverage of the Role ontology was somewhat low since the aim during its development was to capture all the possible concepts and their different relationships even if they were not going to be directly used in the prototype.

\section{Related Work}

Different proposals have been made for designing sensor network infrastructures to integrate data coming from different sources. Sensor network infrastructures follow different approaches to represent data ${ }^{2}$; some of them use database models, e.g., SenseWeb (Kansal, Nath, Liu, \& Zhao, 2007); others use XML schemas IrisNet (Gibbons, Karp, Ke, Nath, \& Seshan, 2003), Hourglass (Shneidman et al., 2004), SemSOS (Henson, Pschorr, Sheth, \& Thirunarayan, 2009), and SANY (Klopfer \& Simonis, 2010); and some others use ontological models REASON (Jabeur, McCarthy, Xing, \& Graniero, 2009) and SWAP (Moodley, 2009).

Knowing the concrete schemas that these infrastructures use to represent their data is not apparent in the literature because scarce details on these schemas are provided and because their definitions are not available. Even if this lack of information may be due to the fact that these models are considered as implementationspecific information, our viewpoint is that having details about such models can facilitate data reuse and interoperability.

Similarly to our approach, other existing sensor network infrastructures manage data to represent sensor networks and their spatio-temporal characteristics, including information to support the discovery and integration of sensor information (mainly through registries). However, no data model has been proposed yet for representing every type of data required by the current Semantic Sensor Web infrastructures.

Depending on the intended use of the infrastructures, some types of data are covered or not; for example, dataset descriptions are only used in SANY, SemSOS, SenseWeb, and SWAP, whereas service descriptions are only used in Hourglass SANY, and SWAP.

One type of data that we have not found in these infrastructures, and that we take into account in our model, is that about the schemas used in the different data sources to represent their underlying data, which are needed for integrating data from heterogeneous data sources (e.g., sensor data streams, relational databases or XML data). In the infrastructures mentioned, this integration is performed by different components responsible for converting data to a common model. However, in our case integration is managed by a specific integration service that requires information about the underlying data schemas (Calbimonte, Corcho, \& Gray, 2010).

Sensor network infrastructures have been extensively applied to environmental monitoring (Hart \& Martinez, 2006), mainly in the field of risk monitoring and management; these infrastructures have been used in different domains such as volcanic eruptions (WernerAllen, Johnson, Ruiz, Lees, \& Welsh, 2005), forest fires (Terhorst et al., 2006), agricultural risks (Panchard, Rao, Prabhakar, Hubaux, \& Jamadagni, 2007), or flood events (Baptista, 2006; Chang \& Guo, 2006; Zhou \& De Roure, 2007; Smith et al., 2009; Sunkpho \& Ootamakorn, 2011), which is our case. 
The use of ontologies to model domain data in these infrastructures is quite recent (Asunción et al., 2005; Tanasescu et al., 2006; Terhorst et al., 2006; McCarthy et al., 2008; Klopfer \& Simonis, 2010), and the models defined in each infrastructure are different and difficult to reuse. This is mainly so because the use cases implemented over these sensing infrastructures are quite heterogeneous and their end users work under aset of specific regulations (e.g., theFlood and Water Management Act 2010 in the UK, which is our case, or the Andalusian Regional Ministry of Environment in Spain) (Asunción et al., 2005) that are completely different from one scenario to another.

Nevertheless, the principles for modelling domain-specific features of interest and properties in these infrastructures are similar and, since the appearance of the Observations and Measurements model (Cox et al., 2010), all the approaches are based on it. All these models usually include feature and property categories (i.e., hierarchies), and the only noticeable features in them are the absence of the rich expressivity that the ontology language permits and of the representation of links between feature categories and properties. These absences can be justified by the lack of requirements that demand them in the corresponding use scenarios; however, we have also included recommendations for them in the guidelines presented.

Another remarkable fact is that only one of these infrastructures has reused existing ontologies during development (the AFIS system reuses the SWEET ontologies) (Terhorst et al., 2006). In our opinion, the development of models for Semantic Sensor Network infrastructures should be guided by reuse, which has been our approach while developing the ontologies presented in this paper. We think that the Semantic Sensor Network ontology proposed in the W3C (Lefort et al., 2011) will fill an important gap here by allowing the interchange of cross-domain sensor data between infrastructures.

\section{CONCLUSION}

This paper presents an ontological model that can be fully or partially reused in Semantic Sensor Web infrastructures; by reusing upper ontologies, we aim to facilitate interoperability between such infrastructures. Additionally, the separation between the infrastructure and the domain ontologies contributes to making the ontological model modular and extensible.

The use of the ontological model in a different domain or use case should not entail major problems, since our design principles have been to reuse ontologies and data models as much as possible and to modularize the ontologies developed so as to facilitate the reuse or replacement of ontology modules.

While some of the ontologies are specific to the coastal flood warning scenario (e.g., Role), those related to the infrastructure are generic. Thus, the ontological model can be adapted to other situations by replacing the domain ontologies. Note that if an ontology exists for the new application domain, then to replace the domain ontologies with an ontology for the new domainis a straightforward process. Otherwise, a new ontology would have to be developed. This development requires a domainexpert who knows the terminology and relationships of the concepts in the domain who in turn should work with an ontology engineer.

In the paper we present a static evaluation of the ontologies developed. Clearly, a complete evaluation would also require performing dynamic evaluations over a running system using the ontologies (e.g., to analyze the effect of the ontology structure in query complexity or efficiency).

The evaluation results unveiled some problems (or potential ones) in the ontologies that were later fixed. It is also important to remark the important role of evaluation in the ontology development process since sometimes problems in the ontologies may not arise from the same process but from other factors, such as changes in the ontologies reused, as it occurred in our case. 
However, ontology evaluation is not a straightforward task. Even if different ontology evaluation methods are proposed in the literature, applying them requires manual intervention minimally supported by software tools. Therefore, we would like to remark the need for automation of ontology evaluation activities by means of software ontology evaluation frameworks that facilitate evaluation and enable advanced evaluation scenarios (e.g., regression evaluations).

Furthermore, existing ontology evaluation techniques are domain independent; however, it would also be desirable to have domainspecific ontology evaluation techniques and guidelines. An example of such a technique in the domain of sensor networks would be one that allows evaluating whether the SSN ontology is properly being used in those ontologies that reuse or extend it.

\section{ACKNOWLEDGMENT}

This work has been supported by the SemSorGrid4Env European project (FP7-223913) and by the Spanish project myBigData (TIN201017060). Thanks to Jean-Paul Calbimonte, Alex Frazer, Alasdair J.G. Gray, Kostis Kyzirakos, Kevin Page, Jason Sadler, and the rest of the people in the SemSorGrid4Env project for providing input and feedback on the ontologies. Thanks to Rosario Plaza for reviewing the grammar of this paper.

\section{REFERENCES}

Asunción, M.,dela, Castillo,L.,Fernández-Olivares, J., García-Pérez, O., González,A., \& Palao, F. (2005). SIADEX: An interactive knowledge-based planner for decision support in forest fire fighting. $A I \mathrm{Com}$ munications, 18(4), 257-268.

Baptista, A. (2006, September). CORIE: The first decade of a coastal-margin collaborative observatory. In Proceedings of the MTS/IEEE Conference for Ocean Engineering, Science and Technology (pp. 1-6).
Bartha, M., Bleier, T., Dihé, P., Havlik, D., Hilbring, D., \& Hugentobler, M. ...Watson, K. (2009). Specification of the Sensor Service Architecture (Sensor $S A$ ) (OGC Discussion Paper 09-132r1). Wayland, MA: Open Geospatial Consortium

Bechhofer, S., \& Volz, R. (2003). WonderWeb OWL ontology validator. Retrieved from http://www. mygrid.org.uk/OWL/Validator

Berners-Lee, T. (2006). Linked data. Retrieved from http://www.w3.org/DesignIssues/LinkedData. htm1Botts

Berrueta, D., \& Polo, L. (2008). Morfeo project: Measurement units ontology. Retrieved from http://forge.morfeo-project.org/wiki_en/index.php/ Units_of_measurement_ontology

Botts, M., \& Robin, A. (2007). OpenGIS Sensor Model Language (SensorML) v1.0.0 (Tech. Rep. No. 07-000). Wayland, MA: Open Geospatial Consortium.

Brank, J., Grobelnik, M., \& Mladenić, D. (2005). A survey of ontology evaluation techniques. In Proceedings of the 8th International Multi-conference of the Information Society, Ljubljana, Slovenia (pp. 166-169).

Brewster, C., Alani, H., Dasmahapatra, S., \& Wilks, Y. (2004). Data-driven ontology evaluation. In Proceedings of the Language Resources and Evaluation Conference, Lisbon, Portugal (pp. 164-168).

Calbimonte, J.-P., Corcho, O., \& Gray, A. J. G. (2010, November). Enabling ontology-based access to streaming data sources. In Proceedings of the 9th International Semantic Web Conference, Shanghai, China (pp. 96-111).

Chang, N.-B., \& Guo, D.-H. (2006, April). Urban flash flood monitoring, mapping and forecasting via a tailored sensor network system. In Proceedings of the IEEE International Conference on Networking, Sensing and Control, Ft. Lauderdale, FL (pp. 757-761).

Cox, S., Botts, M., Robin, A., Atkinson, R., Falke, S., \& Woolf, A. ...Lawrence, B. (2010). Geographic information: Observations and measurements. OGC abstract specification topic 20 (Tech. Rep. No. 10004r3). Wayland, MA: Open GeospatialConsortium.

Davidson, J. (2006, October). OGC Sensor Web enablement: Overview and high level architecture. In Proceedings of the 2nd International Conference on Geosensor Networks (pp. 175-190). 
Digital Equipment Corporation. (1992). Information technology - Database language SQL. Retrieved from http://www.contrib.andrew.cmu.edu/ shadow/ $\mathrm{sql} / \mathrm{sql1992.txt}$

Gangemi,A., Catenacci,C.,Ciaramita, M., \&Lehmann, J. (2005). A theoretical framework for ontology evaluation and validation. In Proceedings of the 2 nd Italian Semantic Web Workshop, Trento, Italy.

Gibbons, P. B., Karp, B., Ke, Y., Nath, S., \& Seshan, S. (2003). IrisNet: An architecture for a worldwide sensor web. IEEE Pervasive Computing, 2(4), 22-33. doi:10.1109/MPRV.2003.1251166

Gómez-Pérez,A., Martinez, K., Fernandes, A.A., \& Ibarrola, A. (2008). Index of ontologies. Retrieved from http://www.semsorgrid4env.eu/ontologies/

Gray, A. J. G., Sadler, J., Kit, O., Kyzirakos, K., Karpathiotakis, M., \& Calbimonte, J.-P. (2011). A semantic sensor web for environmental decision support applications. Sensors, 11(9), 8855-8887. doi: $10.3390 / \mathrm{s} 110908855$

Guarino,N., \&Welty, C. (2002). Evaluating ontological decisions with OntoClean. Communications of the ACM, 45(2),61-65. doi:10.1145/503124.503150

Hart, J., \& Martinez, K. (2006). Environmental sensor networks: A revolution in the earth system science? Earth-Science Reviews, 78(3-4), 177-191. doi:10.1016/j.earscirev.2006.05.001

Henson, C., Pschorr, J., Sheth, A., \& Thirunarayan, K. (2009, May). SemSOS: Semantic sensor observation service. In Proceedings of the International Symposium on Collaborative Technologies and Systems, Baltimore, MD (pp. 44-53).

ISO/IEC. (2005a). Geographic information-Rules for application schema. Geneva, Switzerland: ISO/ IEC.

ISO/IEC. (2005b). Geographic information - Services. Geneva, Switzerland: ISO/IEC.

Jabeur, N., McCarthy, J., Xing, X., \& Graniero, P. (2009). A knowledge-oriented meta-framework for integrating sensor network infrastructures. Computers \& Geosciences, 35(4), 809-819. doi:10.1016/j. cageo.2008.04.006

Kansal, A., Nath, S., Liu, J., \& Zhao, F. (2007). SenseWeb: An infrastructure for shared sensing. IEEE MultiMedia, I4(4), 8-13. doi: 10.1109/ MMUL.2007.82

Klopfer, M., \& Simonis, I. (Eds.). (2010). SANY An open service architecture for sensor networks. Hunan, China: The SANY Consortium.
Klyne, G., \& Carroll, J. J. (2004). Resource Description Framework $(R D F)$ : Concepts andabstractsyntax (Tech. Rep.). Retrieved from http://www.w3.org/ TR/rdf-concepts/

Lefort, L., Henson, C., Taylor, K., Barnaghi, P., Compton, M., \& Corcho, O. ...Page, K. (2011). Semantic sensor network XG final report (Tech. Rep.). Retrieved from http://www.w3.org/2005/Incubator/ ssn/XGR-ssn-20110628/

Lefort, L., Sheth,A., \& Taylor, K. (2008). Incubator activity: W3C semantic sensor network incubator group. Retrieved from http://www.w3.org/2005/ Incubator/ssn/

Maynard, D., \& Harth, A. (2012). Semantic Web challenges. Retrieved from http://challenge.semanticweb.org/

McCarthy, J., Graniero, P., \& Rozic, S. (2008). An integrated GIS-expert system framework for live hazard monitoring and detection. Sensors, 8 , 830-846. doi: $10.3390 / \mathrm{s} 8020830$

McGuiness, D., \& van Harmelen, F. (2004). OWL Web ontologylanguage overview (Tech. Rep.). Retrieved from http://www.w3.org/TR/owl-features/

Moodley, D. (2009). Ontology driven multi-agent systems: An architecture for sensor web applications (Unpublished doctoral dissertation). School of Computer Science, University of KwaZulu-Natal, KwaZulu-Natal, South Africa.

Obrst, L., Ashpole, B., Ceusters, W., Mani, I., Ray, S., \& Smith, B. (2007). The evaluation of ontologies - Toward improved semantic interoperability. In Baker, C. J. O., \& Cheung, K.-H. (Eds.), Semantic Web: Revolutionizing knowledge discovery in the life sciences (pp. 139-158). New York, NY: Springer.

OGC. (2012). Geospatial and location standards. Retrieved from http://www.opengeospatial.org/

OGC.(n.d.). Sensor Web enablementDWG. Retrieved from http://www.opengeospatial.org/projects/ groups/sensorwebdwg

Page, K., De Roure, D., Martinez, K., Sadler, J. D., $\&$ Kit, O. Y. (2009, October). Linked sensor data: RESTfully Serving RDF and GML. InProceedings of the 2nd International Workshop on Semantic Sensor Networks, Washington, DC (pp. 49-63).

Pak, J.,\&Zhou,L. (2010). A framework for ontology evaluation. In Proceedings of the 8th Workshop on eBusiness, Phoenix, AZ (pp. 10-18). 
Panchard, J., Rao, S., Prabhakar, T., Hubaux, J.-P., \& Jamadagni, H. (2007). COMMONSense Net: A wireless sensor network for resource-poor agriculture in the semiarid areas of developing countries. Information Technologies and International Development, 4(1), 51-67. doi:10.1162/itid.2007.4.1.51

Pérez de Laborda, C., \& Conrad, S. (2005, January). Relational.OWL: A data and schema representation format based on OWL. In Proceedings of the 2nd Asia-Pacific Conference on Conceptual Modelling, Newcastle, Australia (pp. 89-96).

Prud'hommeaux, E. (2007). RDF validation service. Retrieved from http://www.w3.org/RDF/Validator/

Raskin, R. G., \& Pan, M. J. (2005). Knowledge representation in the Semantic Web for Earth and environmental terminology (SWEET). Computers \& Geosciences, 31, 1119-1125. doi:10.1016/j. cageo.2004.12.004

RSS Advisory Board. (2009). RSS 2.0 specification. Retrieved from http://www.rssboard.org/ rss-specification

Sheth,A., Henson, C., \& Sahoo, S. (2008). Semantic SensorWeb. IEEE Internet Computing, 12(4), 78-83. doi: 10.1109/MIC.2008.87

Shneidman, J.,Pietzuch, P., Ledlie, J., Roussopoulos, M., Seltzer, M., \& Welsh, M. (2004). Hourglass: An infrastructure for connecting sensor networks and applications (Tech. Rep.). Boston, MA: Harvard University.

Smith, P. J., Hughes, D., Beven, K. J., Cross, P., Tych, W., Coulson, G., \& Blair, G. (2009). Towards the provision of site specific flood warnings using wireless sensor networks. Meteorological Applications, 16(1), 57-64. doi:10.1002/met.130

Sunkpho, J., \& Ootamakorn, C. (2011). Real-time flood monitoring and warning system. Songklanakarin Journal of Science and Technology, 33(2), 227-235.
Tanasescu, V., Gugliotta,A., Domingue, J., GutierrezVillarias, L., Davies, R., Rowlatt, M., \& Richardson, M. (2006, June). A semantic Web GIS based emergency management system. In Proceedings of the International Workshop on Semantic Web for E-government, Budva, Montenegro (pp. 1-12).

Terhorst, A., Moodley, D., Simonis, I., Frost, P., McFerren, G., Roos, S., \& van den Bergh, F. (2006, October). Using the SensorWeb to detect andmonitor the spread of vegetation fires in southern Africa. In Proceedings of the Second International Conference on Geosensor Networks, Boston, MA (pp. 239-251).

UK Public General Act, Flood and Water Management Act 2010, c.29. (2010, April).

United States Department of Homeland Security. (n.d.).U.S. CoastGuardnavigationcenter. Retrieved from http://www.navcen.uscg.gov/?pageName=AIS

Vrandečić, D. (2010). Ontology evaluation (Unpublished doctoral dissertation). KIT, Fakultät für Wirtschaftswissenschaften, Karlsruhe, Germany.

Werner-Allen, G., Johnson, J., Ruiz, M., Lees, J., \& Welsh, M. (2005, January 31-February 2). Monitoring volcanic eruptions with a wireless sensor network. In Proceedings of the 2nd European Workshop on Wireless Sensor Networks (pp. 108-120).

Zhou, J.,\&DeRoure,D. (2007). FloodNet: Coupling adaptive sampling with energy aware routing in a flood warning system. Joumal of Computer Science and Technology, 22(1), 121-130. doi:10.1007/ s11390-007-9017-7

Zolin, E. (2011). Complexity of reasoning in description logics. Retrieved from http://www.cs.man. ac.uk/ ezolin/dl/

\section{ENDNOTES}

1 The names of the ontologies have been shortened: Additional Regions $=$ AR, Coastal Defences $=\mathrm{CD}$, Role $=$ RO, Schema $=\mathrm{SM}$, Service $=$ SO, SSN Extension $=$ SS

2 because it is out of the scope of this paper.

Copyright (C) 2012, IGI Global. Copying or distributing in print or electronic forms without written permission of IGI Global is prohibited. 
Raúl Garcia-Castro is a postdoctoral researcher at the Ontology Engineering Group, Universidad Politécnica de Madrid (UPM), Spain. His research focuses on the evaluation of semantic technologies and on ontology engineering and has been published in a number of academic journals, conference publications, and books. In 2008 he obtained a PhD in Computer Science and Artificial Intelligence at the UPM with his thesis titled "Benchmarking Semantic Web Technology," which obtained the PhD Extraordinary Award. He regularly participates in the program committees of the conferences and workshops that are most relevant in the area and has participated in the organization of several of them.

Oscar Corcho is an Associate Professor at Departamento de Inteligencia Artificial (Facultad de Informática, Universidad Politécnica de Madrid), and he belongs to the Ontology Engineering Group. His research activities are focused on Semantic e-Science and Real World Internet, although he also works in the more general areas of Semantic Web and Ontological Engineering. He has participated in a number of EU projects (Wf4Ever, PlanetData, SemsorGrid4Env, ADMIRE, OntoGrid, Esperonto, Knowledge Web and OntoWeb), and Spanish R\&D projects, and has also participated in privately-funded projects like ICPS, funded by the World Health Organisation, and HALO, funded by Vulcan Inc. Previously, he worked as a Marie Curie research fellow at the University of Manchester, and was a research manager at iSOCO. He holds a degree in Computer Science, an MSc in Software Engineering and a PhD in Computer Science and Artificial Intelligence from UPM. He was awarded the Third National Award by the Spanish Ministry of Education in 2001. He has published several books, from which "Ontological Engineering" can be highlighted as it is being used as a reference book in a good number of university lectures worldwide, and more than 100 papers in journals, conferences and workshops.

Chris Hill is Director of the GeoData Institute, an interdisciplinary research group at the Southampton University, UK. The group provides environmental data and information applied research that crosses the physical and social disciplines. His research interests lie in geospatial data and information for biodiversity and habitat analyses, natural resource management and environmental decision support. Recent work focuses on spatial data infrastructures in the marine and coastal environment and the generation of information frameworks for vulnerability and risk assessment. 